\title{
Immunochemical Identification of Distinct Molecular Species of Collagen in the Squid Muscle and Skin
}

\author{
Shohshi Mizuta, ${ }^{* 1}$ Reiji Yoshinaka, ${ }^{* 1}$ Mamoru Sato, ${ }^{* 2}$ \\ and Morihiko Sakaguchi*3 \\ *1 Department of Marine Bioscience, Faculty of Biotechnology, Fukui Prefectural University, \\ Obama, Fukui 917, Japan \\ ${ }^{* 2}$ Faculty of Fisheries, Kagoshima University, Shimoarata, Kagoshima 890, Japan \\ ${ }^{* 3}$ Department of Fisheries, Faculty of Agriculture, Kyoto University, Sakyo, Kyoto 606-01, Japan
}

(Received March 15, 1995)

Two genetically distinct types of collagen, Type SQ-I and SQ-II, were isolated from the mantle muscle of the squid Todarodes pacificus, and their respective components, $\alpha 1$ (SQ-I) and $\alpha 1$ (SQ-II), were resolved from the two types of collagen by column chromatography. Rabbit antisera against these collagens and $\alpha$ components were prepared and their immunological properties were examined by immunoblot analysis. The anti-Type SQ-I collagen serum reacted intensely to the $\alpha 2$ (SQ-I) and $\alpha 2$ (SQ-II) components but faintly to the $\alpha 1$ (SQ-I) and $\alpha 1$ (SQ-II) components. The anti-Type SQ-II collagen serum reacted intensely to the $\alpha$ (SQ-II) component but showed little reactivity to the other $\alpha$ components. Although the anti- $\alpha 1$ (SQ-I) component serum was rather crossreactive for the $\alpha 1$ (SQ-II) component, it showed a high specificity to the $\alpha 1$ (SQ-I) component by treatment with 20 or $50 \mu \mathrm{g}$ of Type SQII collagen $/ 1 \mu \mathrm{l}$ of serum at $37^{\circ} \mathrm{C}$ for $48 \mathrm{~h}$. On the contrary, the anti- $\alpha 1$ (SQ-II) component serum displayed a high specificity to the homologous antigen. These results suggest that the anti- $\alpha 1$ (SQ-I) and anti- $\alpha$ l(SQ-II) component sera can be applied to immunohistochemical experiments as antibodies which recognize Type SQ-I and SQ-II collagens, respectively. The distribution of these two molecular species of collagen in the mantle skin and arm muscle was also examined by immunoblot analysis. Type SQ-I and SQ-II collagen fractions from the mantle skin and arm muscle reacted to these four antisera in the same way as those prepared from the mantle muscle. These results demonstrate the wide distribution of these two types of collagen not only in the mantle muscle but also in the mantle skin and arm muscle.

Key words: collagen, squid, muscle, mantle, skin, arm, mollusc, molecular species

In vertebrate animals, especially in fish, collagen has recently received increased attention as one of the most important proteins for the expression of meat texture. Fish meat becomes tender and loses firmness during chilled storage. It has been suggested that this phenomenon of meat tenderization is due to the disintegration of collagen fibers which is caused by an enzymatic degradation, probably by the endogenous proteolytic enzymes, of a specific molecular species of collagen, Type $\mathrm{V}^{1-6)}$ This enzymatic degradation of Type $V$ collagen has been suggested by the increased solubility of Type $\mathrm{V}$ collagen during chilled storage of fish meat. ${ }^{5,6)}$ In invertebrate animals, however, only limited information is available concerning the contribution of collagen to meat texture, on abalone, ${ }^{7,8)}$ turban shell, ${ }^{97}$ and several species of crustacean. ${ }^{10)}$

We previously reported the biochemical characteristics of two genetically distinct types of collagen, named Type SQ-I and SQ-II collagens, from the mantle muscle and skin of the squid Todarodes pacificus for the purpose of clarifying the contribution of each molecular species of collagen to meat texture. ${ }^{11,12)}$ For this purpose, it seems to be very important to localize each collagen type in muscular tissues. In this context, the present study was designed to prepare the antisera against distinct molecular species of collagen, and examine the immunological properties of these antisera, aiming at an application for immunohistochemical studies. In addition, the distribution of each molecular species of collagen in the mantle skin and arm muscle was also examined by immunochemical techniques.

\section{Materials and Methods}

\section{Preparation of Collagens and $\alpha$ Chains}

Pepsin-solubilized collagen was prepared from the muscle and skin of the mantle, and arm muscle of the squid Todarodes pacificus (average body weight, $274.9 \mathrm{~g}$ ) and fractionated by $0.45 \mathrm{M} \mathrm{NaCl}$ precipitation into two collagen fractions, the precipitable fraction (Type SQ-I) and the soluble fraction (Type SQ-II), according to the method described previously. ${ }^{11)}$ Moreover, Type SQ-I and SQ-II collagens were purified by phosphocellulose P-11 (Whatman, Maidstone, England) column chromatography from the respective fractions of the mantle muscle as described in the same report. ${ }^{11}$

Two alpha components, $\alpha 1$ (SQ-I) and $\alpha 1$ (SQ-II), were purified from Type SQ-I and SQ-II collagens of the mantle muscle by phosphocellulose column chromatography and gel filtration according to the method in the previous report. ${ }^{12)}$ The collagens were dissolved at a concentration 
of $2 \mathrm{mg} / \mathrm{ml}$ in $20 \mathrm{~mm}$ sodium phosphate, $\mathrm{pH} 6.8$, containing 0 or $50 \mathrm{mM} \mathrm{NaCl}$, denatured by heating at $40^{\circ} \mathrm{C}$ for 10 min, and applied to a column $(1.5 \times 10 \mathrm{~cm})$ of phosphocellulose. Elution was achieved by using the starting buffer with a linear gradient of $0-200$ or $50-250 \mathrm{mM} \mathrm{NaCl}$ over a total volume of $300 \mathrm{ml}$ at a flow rate of $100 \mathrm{ml} / \mathrm{h}$. The effluent was monitored at $230 \mathrm{~nm}$ by a spectrophotometer UV-1200 (Shimadzu, Kyoto, Japan). Appropriate fractions were pooled, dialyzed against distilled water, and lyophilized. The collagen chains from the phosphocellulose column were further resolved by gel filtration. The collagen chains were dissolved in $0.5 \mathrm{ml}$ of $50 \mathrm{mM}$ Tris- $\mathrm{HCl}$, $\mathrm{pH} 7.5$, containing $2 \mathrm{M}$ urea, $0.1 \mathrm{M} \mathrm{NaCl}$, and $0.02 \%$ sodium azide. The sample solution was applied to a column $(1.6 \times 60 \mathrm{~cm}$ ) of Sephacryl S-400 HR (Pharmacia Biotech, Tokyo, Japan) and eluted at a flow rate of $50 \mathrm{ml} / \mathrm{h}$ with $50 \mathrm{~mm}$ Tris- $\mathrm{HCl}$, pH 7.5, containing $0.1 \mathrm{M} \mathrm{NaCl}$. Fractions of $2.0 \mathrm{~m} l$ were collected and monitored at $230 \mathrm{~nm}$. After analysis by sodium dodecyl sulfate-polyacrylamide gel electrophoresis (SDS-PAGE), appropriate fractions were pooled, dialyzed against distilled water, and lyophilized.

These collagen molecules and $\alpha$ components which were chromatographically purified were used as the antigens in the following procedures of antisera preparation.

\section{Preparation of Antisera}

Type SQ-I and SQ-II collagens, and the $\alpha 1$ (SQ-I) and $\alpha 1$ (SQ-II) components from the mantle muscle were dissolved in $0.1 \mathrm{M}$ acetic acid or distilled water at a concentration of $1 \mathrm{mg} / \mathrm{ml}$. The solutions were emulsified in equal volumes of Freund's complete adjuvant. The emulsion $(0.5 \mathrm{ml})$ was injected subcutaneously into the back of rabbits. After 7 and 20 days the rabbits were further immunized with $0.5 \mathrm{ml}$ of the same antigens. The rabbits were boosted in the same way as above at 7 days prior to the collection of blood. Blood was collected from the ear vein and left overnight in a cold room at $5^{\circ} \mathrm{C}$. After centrifugation, the resultant supernatant was used as the antiserum preparation.

\section{SDS-PAGE and Peptide Mapping}

SDS-PAGE was performed by the method of Laemm$1 i^{13)}$ using $5 \%$ gel. Gels were stained for protein with Coomassie Brilliant Blue R-250 according to the method of Fairbanks et al. ${ }^{14)}$ The collagen and related peptides were stained metachromatically and non-collagenous proteins were stained orthochromatically. ${ }^{15,16)}$

Peptide mapping with Achromobactor lyticus lysyl endopeptidase (EC 3.4.21.50, Wako, Osaka, Japan) was performed essentially as described by Cleveland et al. ${ }^{17}$ ) Proteins were denatured in $50 \mathrm{mM}$ Tris- $\mathrm{HCl}, \mathrm{pH} \mathrm{7.5}$, containing $1 \%$ SDS and $30 \%$ glycerol. The sample was applied to the sample well and digested with the protease at an enzyme/substrate weight ratio of 1:20 in the stacking gel. The digest was separated on $10 \%$ gel and stained by the same method as described above.

\section{Immunochemical Analysis}

After separation by SDS-PAGE, collagen chains were transferred to nitrocellulose membranes according to the method of Towbin et al., ${ }^{18)}$ and the membranes were immunostained by the avidin-biotin complex method essentially as previously described. ${ }^{19)}$ The nitrocellulose membranes were blocked with $2 \%$ hen egg ovalbumin in phosphate buffered saline (PBS; $10 \mathrm{~mm}$ sodium phosphate buffer, $\mathrm{pH} 7.4$, containing $150 \mathrm{mM} \mathrm{NaCl}$ ) and then reacted with each antiserum diluted in 1:100-1:1000 in PBS. The biotinylated goat anti-rabbit IgG antibody (Vector Lab., Burlingame, USA) was diluted in 1:1000-1:250 in PBS and used as the secondary antibody. The membranes were then reacted with the avidin-biotin complex which had been prepared by mixing avidin and biotinylated horseradish peroxidase. The binding of antibodies was visualized using $0.04 \%(w / v)$ diaminobenzidine tetrahydrochloride (DAB; Nacalai Tesque, Kyoto, Japan), or $0.06 \%$ 4-chloro-1naphthol (Nacalai Tesque), containing $0.006 \% \mathrm{H}_{2} \mathrm{O}_{2}$. In SDS-PAGE and western blotting, Type SQ-I and SQ-II
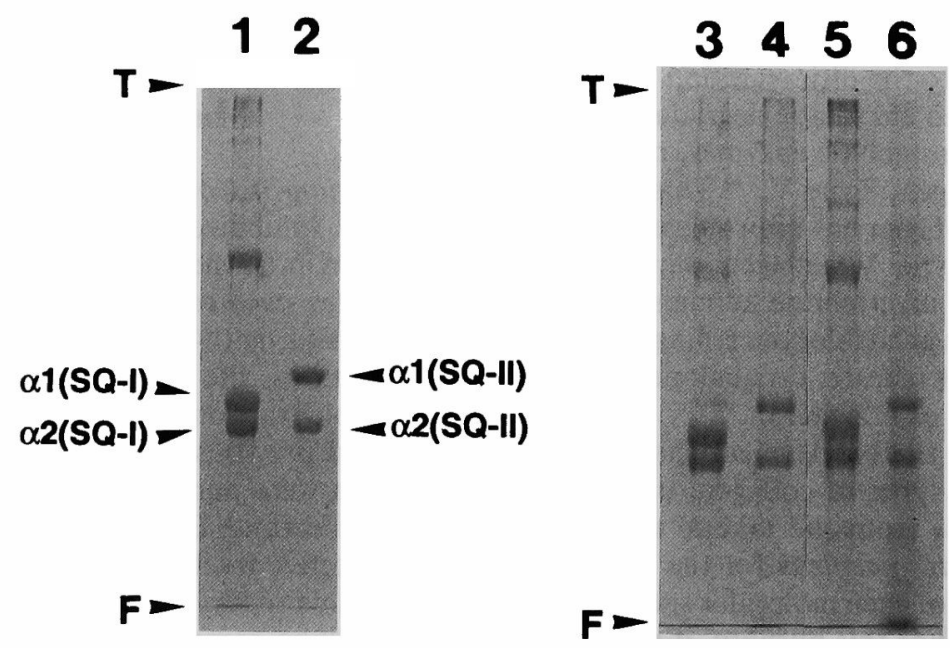

Fig. 1. SDS-PAGE patterns (5\% gel) of Type SQ-I (lanes 1, 3, and 5) and SQ-II (lanes 2, 4, and 6) collagen fractions from the squid mantle muscle (lanes 1 and 2), mantle skin (lanes 3 and 4), and arm muscle (lanes 5 and 6).

Arrows $\alpha 1$ (SQ-I), $\alpha 2$ (SQ-I), $\alpha 1$ (SQ-II), and $\alpha 2$ (SQ-II) show the positions of these components. The letters $\mathrm{T}$ and $\mathrm{F}$ show the top and buffer front of the gel, respectively. 
collagen fractions, which were fractionated only by $\mathrm{NaCl}$ precipitation, were used as the samples.

\section{Results and Discussion}

Lanes 1 and 2 in Fig. 1 show the SDS-PAGE patterns of Type SQ-I and SQ-II collagens from the squid mantle muscle, respectively. As previously reported, ${ }^{11)}$ Type SQ-I collagen contained two bands of $\alpha$ component, termed $\alpha 1$ (SQ-I) and $\alpha 2$ (SQ-I), together with some higher molecular weight components, while Type SQ-II collagen contained two bands of $\alpha$ component, termed $\alpha 1$ (SQ-II) and $\alpha 2$ (SQ-II). In addition, these collagen fractions from the mantle skin showed identical patterns of SDS-PAGE to those from the mantle muscle as described in the previous report (Fig. 1, lanes 3 and 4) ${ }^{11}$ In the case of the arm muscle, Type SQ-I and SQ-II collagen fractions exhibited quite similar patterns to those from the mantle muscle and skin, although several bands of degradation products were observed in the patterns of the Type SQ-II collagen fraction (Fig. 1, lanes 5 and 6). Fig. 2 shows the immnoblot analysis of Type SQ-I and SQ-II collagens stained with the antisera against collagens prepared from the mantle muscle. The anti-Type SQ-I collagen serum reacted strongly to both $\alpha 2$ (SQ-I) and $\alpha 2$ (SQ-II) components with an approximately equal intensity to each other. However, the reactivity to the $\alpha 1$ (SQ-I) and $\alpha 1$ (SQ-II) components was very low (Fig. 2A and 2B). These results support the previous

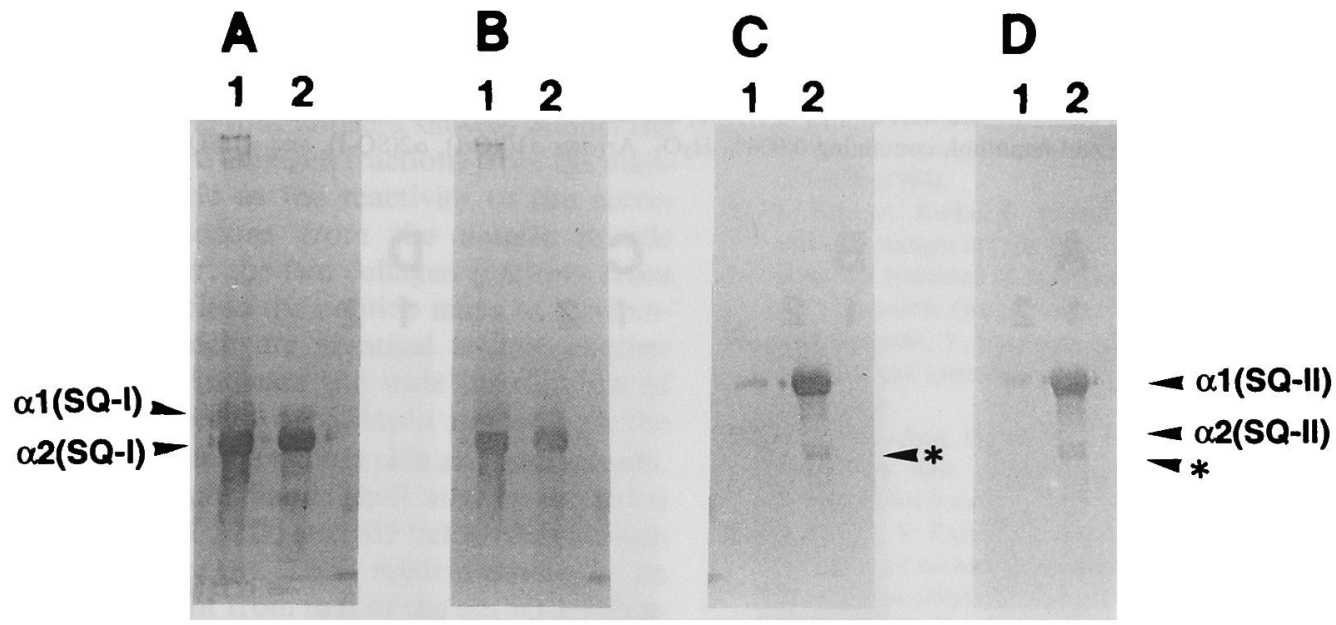

Fig. 2. Immunoblot analysis of Type SQ-I (lane 1) and SQ-II (lane 2) collagen fractions from the mantle muscle stained with the anti-Type SQ-I collagen serum (A and $B$ ) and with the anti-Type SQ-II collagen serum (C and D).

The anti-Type SQ-I and SQ-II collagens sera were diluted in 1:500 (A and C) or 1:1000 (B and D), and used as primary antibodies. The binding of the antibodies was visualized with $0.06 \%$ (w/v) of 4-chloro-1-naphthol, containing $0.006 \% \mathrm{H}_{2} \mathrm{O}_{2}$. Arrows $\alpha 1$ (SQ-I), $\alpha 2$ (SQ-I), $\alpha 1$ (SQ-II), and $\alpha 2$ (SQ-II): the same as in Fig. 1.

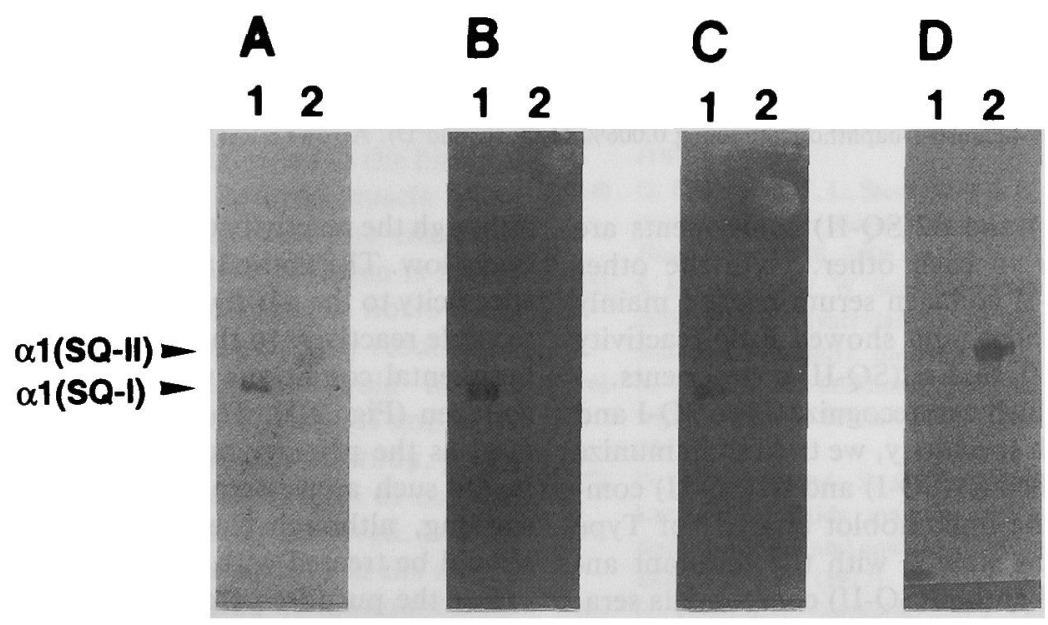

Fig. 3. Immunoblot analysis of Type SQ-I (lane 1) and SQ-II (lane 2) collagen fractions from the mantle muscle stained with the anti- $\alpha$ l(SQ-I) component serum (A, B and C) and with the anti- $\alpha$ I(SQ-II) component serum (D).

The anti- $\alpha 1$ (SQ-I) component (diluted in 1:100) or anti- $\alpha 1$ (SQ-II) component (diluted in 1:1000) serum was used unabsorbed (A and D) or absorbed with 20 (B) and $50 \mu \mathrm{g}$ (C) of Type SQ-II collagen $/ 1 \mu \mathrm{l}$ of serum. The binding of the antibodies was visualized with $0.04 \%$ DAB containing $0.006 \% \mathrm{H}_{2} \mathrm{O}_{2}$ (A, B, and C) or $0.06 \%$ of 4-chloro-1-naphthol containing $0.006 \% \mathrm{H}_{2} \mathrm{O}_{2}$ (D). Arrows $\alpha 1$ (SQ-I) and $\alpha 1$ (SQ-II): the same as in Fig. 1. 


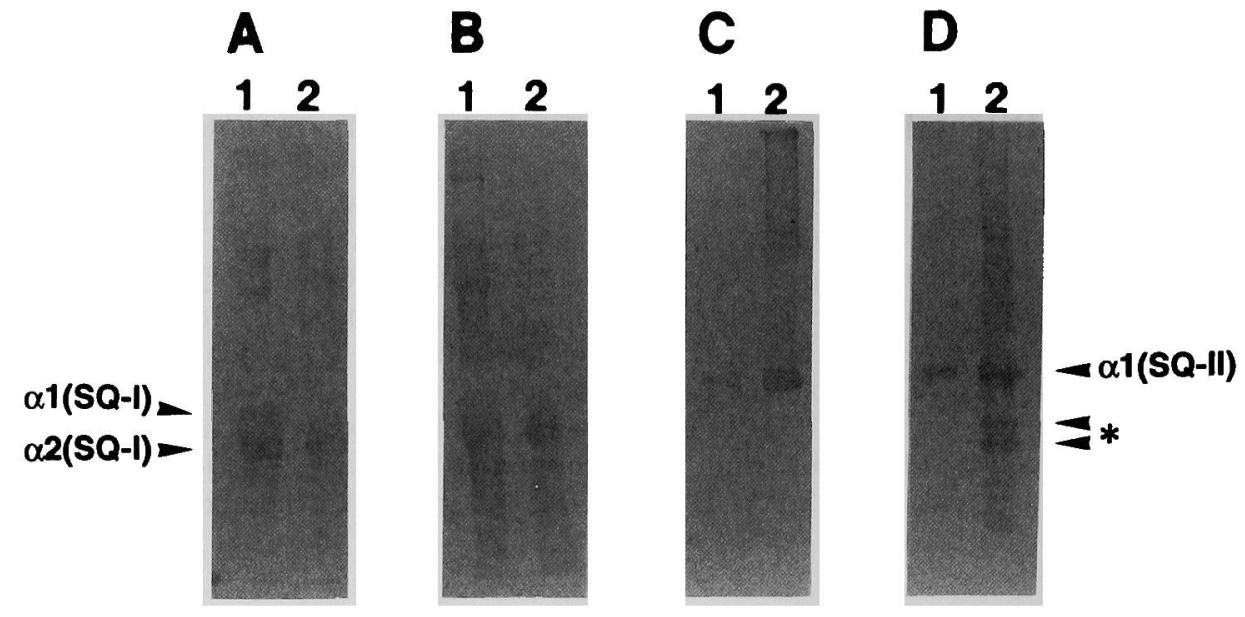

Fig. 4. Immunoblot analysis of Type SQ-I (lane 1) and SQ-II (lane 2) collagen fractions from the mantle skin (A and C) and from the arm muscle (B and D) stained with the anti-SQ-I collagen serum (A and $B$ ) and with the anti-SQ-II collagen serum (C and D).

The anti-Type SQ-I and SQ-II collagens sera were diluted in 1:1000, and used as primary antibodies. The binding of the antibodies was visualized with $0.06 \%(\mathrm{w} / \mathrm{v})$ of 4-chloro-l-naphthol, containing $0.006 \% \mathrm{H}_{2} \mathrm{O}_{2}$. Arrows $\alpha 1(\mathrm{SQ}-\mathrm{I}), \alpha 2(\mathrm{SQ}-\mathrm{I})$, and $\alpha 1$ (SQ-II): the same as in Fig. 1.

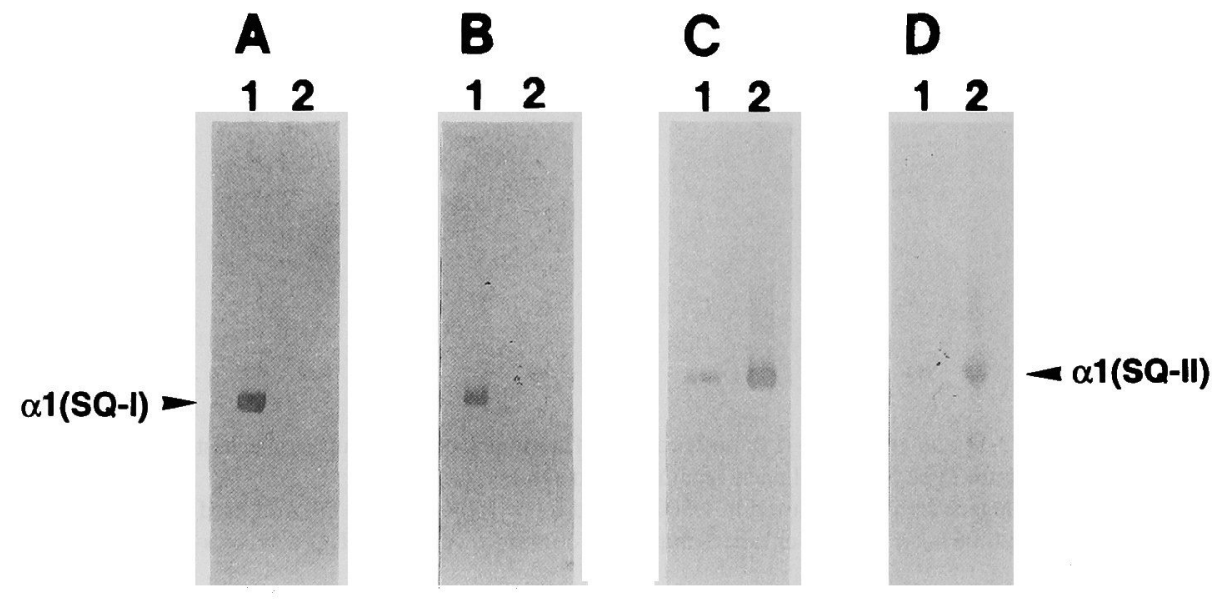

Fig. 5. Immunoblot analysis of Type SQ-I (lane 1) and SQ-II (lane 2) collagen fractions from the mantle skin (A and C) and arm muscle (B and D) stained with the anti- $\alpha 1$ (SQ-I) component serum (A and B) and with the anti- $\alpha 1$ (SQ-II) component serum (C and D).

The anti- $\alpha 1$ (SQ-I) component serum was absorbed with $50 \mu \mathrm{g}$ of Type SQ-II collagen/1 $\mu l$ of serum and diluted in 1:100. The anti- $\alpha 1$ (SQ-II) component serum (diluted in 1:1000) was used unabsorbed. The binding of the antibodies was visualized with $0.04 \%$ DAB containing $0.006 \% \mathrm{H}_{2}$ $\mathrm{O}_{2}$ (A and B) or $0.06 \%$ of 4-chloro-1-naphthol containing $0.006 \% \mathrm{H}_{2} \mathrm{O}_{2}(\mathrm{C}$ and D). Arrows $\alpha 1$ (SQ-I) and $\alpha 1$ (SQ-II): the same as in Fig. 1.

report that the $\alpha 2$ (SQ-1) and $\alpha 2$ (SQ-II) components are structurally very similar to each other. ${ }^{12)}$ On the other hand, the anti-Type SQ-II collagen serum reacted mainly to the $\alpha 1$ (SQ-II) component, and showed little reactivity to the $\alpha 1$ (SQ-I), $\alpha 2$ (SQ-I), and $\alpha 2$ (SQ-II) components.

To prepare antisera which can recognize Type SQ-I and SQ-II collagens with high specificity, we tried to immunize rabbits with highly purified $\alpha 1$ (SQ-I) and $\alpha 1$ (SQ-II) components. Fig. 3 shows the immunoblot analysis of Type SQ-I and SQ-II collagens stained with the resultant antisera, anti- $\alpha 1$ (SQ-I) and anti- $\alpha 1$ (SQ-II) components sera. The anti- $\alpha 1$ (SQ-I) component serum exhibited a major reactivity to the $\alpha 1$ (SQ-I) component and a minor reactivity to the $\alpha 1$ (SQ-II) component (Fig. 3A). The crossreactivity to the $\alpha 1$ (SQ-II) component, however, was almost completely excluded by treatment with 20 or $50 \mu \mathrm{g}$ Type SQ-II collagen $/ 1 \mu l$ of serum for $48 \mathrm{~h}$ at $37^{\circ} \mathrm{C}$ (Fig. 3B and $3 \mathrm{C}$ ), although the reactivity to the homologous antigen was relatively low. The anti- $\alpha$ 1(SQ-II) component had a very high specificity to the $\alpha 1$ (SQ-II) component, and showed no detectable reactivity to the other components even in the experimental conditions without absorbing with Type SQ-I collagen (Fig. 3D). Therefore, these two antisera can be used as the primary antibodies in immunological experiments such as western blotting or immunohistochemical staining, although the anti- $\alpha 1$ (SQ-I) component serum should be treated with Type SQ-II collagen.

For the purpose of clarifying the distrubution of Type SQ-I and SQ-II collagens in the mantle skin and arm muscle, the reactivity of the four antisera described above to the Type SQ-I and SQ-II collagen fractions from the two tissues was examined along with Type SQ-I and SQ-II collagens from the mantle muscle. As shown in Fig. 1, SDSPAGE patterns of the two collagen fractions from the 

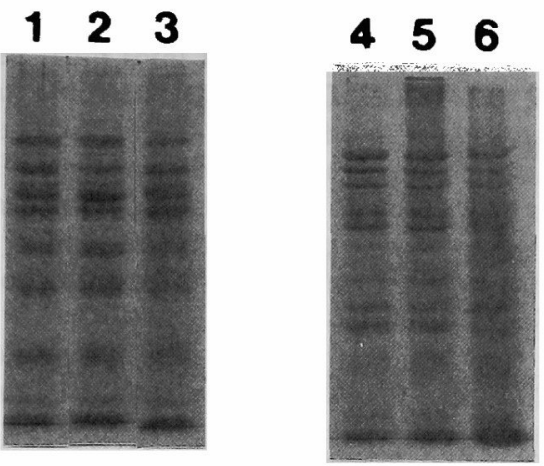

Fig. 6. Peptide maps of the lysyl endopeptidase digests of Type SQ-I (lanes 1, 2, and 3) and SQ-II (Lanes 4, 5, and 6) collagen fractions from the mantle muscle (lanes 1 and 4), mantle skin (lanes 2 and 5), and arm muscle (lanes 3 and 6).

mantle skin and arm muscle were almost identical to those from mantle muscle. The four antisera showed almost the same reactivity to the two collagen fractions from the mantle skin and arm muscle as the reactivity to the corresponding collagen fractions from the mantle muscle (Fig. 4 and 5). Moreover, the two collagen fractions from these three tissues exhibited the peptide maps of lysyl endopeptidase digests which are identical to one another (Fig. 6). These results indicate the wide distribution of these two molecular species of collagen not only in the mantle muscle but also in the mantle skin and arm muscle. Incidentally, several minor bands (indicated by asterisks) were observed in Fig. 2C, 2D, and 4D below the position of the $\alpha$ (SQ-II) component. Their relative mobilities on SDS-PAGE were different from that of the $\alpha 2$ (SQ-II) component. In addition, there was no reproducibility in the relative staining intensity of them to the $\alpha 1$ (SQ-II) component in immunoblot analysis. From these facts, they seemd not to be the $\alpha 2$ (SQ-II) component but to be degradation products derived from the $\alpha 1$ (SQ-II) component.

We have already reported that Type SQ-II collagen has similar biochemical features to Type V collagen in fish muscle. ${ }^{11,12}$ This fact suggests that Type SQ-II collagen may play similar food chemical functions in the squid muscle to those of vertebrate Type $\mathrm{V}$ collagen. Type $\mathrm{V}$ collagen is a fibrous collagen which is mainly distributed in the fine connective tissue, called endomysium, around muscle fibers. In fish species, it has been suggested that the enzymatic degradation of telopeptides of Type $\mathrm{V}$ collagen is mainly responsible for the disintegration of collagen fibers in the endomysium during chilled storage, probably resulting in the meat tenderization. ${ }^{1-6}$ ) As for the squid muscle, however, histological distribution of Type SQ-I and SQ-II collagens remains to be solved. In the present study, preparation of antiserum to each molecular species of collagen from the mantle muscle was successfully achieved. In addition, the existence of these molecular species in the mantle skin and arm muscle was demonstrated by immunochemical technique and peptide mapping. Studies are now in progress to elucidate the histological location of Type SQI and SQ-II collagens in the muscle and skin by appyling immunohistochemical techniques.
Marine Bioscience, Fukui Prefectural University, Fukui, Japan, for his valuable advice.

\section{References}

1) K. Sato, C. Ohashi, K. Ohtsuki, and M. Kawabata: Type V collagen in trout (Salmo gairdneri) muscle and its solubility change during chilled storage of muscle. J. Agric. Food Chem., 39, 1222-1225 (1991).

2) M. Ando, H. Toyohara, Y. Shimizu, and M. Sakaguchi: Post-mortem tenderization of rainbow trout (Oncorhyncus mykiss) muscle caused by gradual disintegration of the extracellular matrix structure. J. Sci. Food Agric., 55, 589-597 (1991).

3) M. Ando, H. Toyohara, and M. Sakaguchi: Post-mortem tenderization of rainbow trout muscle caused by the disintegration of collagen fibers in the pericellular connective tissue. Nippon Suisan Gakkaishi, 58, 567-570(1992).

4) M. Ando, H. Toyohara, Y. Shimizu, and M. Sakaguchi: Post-mortem tenderization of fish muscle due to weakening of pericellular connective tissue. Nippon Suisan Gakkaishi, 59, 1073-1076 (1993).

5) K. Sato, A. Sakuma, K. Ohtsuki, and M. Kawabata: Subunit composition of eel (Anguilla japonica) type V collagen: evidence for existence of a novel fourth $\alpha 4(\mathrm{~V})$ chain. J. Agric. Food Chem., 42, 675-678 (1994).

6) K. Sato, A. Koike, R. Yoshinaka, M. Sato, and Y Shimizu: Postmortem changes in type I and V collagens in myocommatal and endomysial fractions of rainbow trout (Oncorhynchus mykiss) muscle. J. Aquatic Food Product Technol., 3, 5-11 (1994).

7) N. Takayama, Y. Yamanoto, Y. Kadowaki, and K. Endo: Abalone collagen and texture of abalone flesh. Kaseigaku Zasshi, 21, 239242 (1970).

8) R. P. Olaechea, H. Ushio, S. Watabe, K. Takada, and K. Hatae: Toughness and collagen content of abalone muscle. Biosci. Biotech. Biochem., 57, 6-11 (1993).

9) Y. Ochiai, Y. Kariya, S. Watabe, and K. Hashimoto: Heat-induced tendering of turban shell (Batillus cornutus) muscle. J. Food Sci., 50, 981-984 (1985).

10) S. Mizuta, R. Yoshinaka, M. Sato, and M. Sakaguchi: Characterization of collagen in the muscle of several crustacean species in association with raw meat texture. Fisheries Sci., 60, 323-328 (1994).

11) S. Mizuta, R. Yoshinaka. M. Sato, and M. Sakaguchi: Isolation and partial characterization of two distinct types of collagen in the muscle and skin of the squid Todarodes pacificus. Fisheries Sci., 60 , 467-471 (1994).

12) S. Mizuta, R. Yoshinaka. M. Sato, Y. Itoh, and M. Sakaguchi: Subunit composition of two distinct types of collagen in the muscle of the squid Todarodes pacificus. Fisheries Sci., 60, 597-602 (1994).

13) U. K. Laemmli: Cleavage of structural proteins during the assembly of the head of bacteriophage T4. Nature, Lond., 227, 680-685 (1970).

14) G. Fairbanks, T. L. Steck, and D. F. Wallach: Electrophoretic analysis of the major polypeptides of the human erythrocyte membrane. Biochemistry, 10, 2606-2616 (1971).

15) S. Micko and W. W. Schlaepfer: Metachromasy of peripheral nerve collagen on polyacrylamide gel staining with Coomassie Brilliant Blue R-250. Analyt. Biochem., 88, 566-572 (1978).

16) R. C. Duhamel: Differential staining of collagens and non-collagens with Coomassie Brilliant Blue G and R. Collagen Rel. Res., 3, 195 204 (1983).

17) D. W. Cleveland, S. G. Fischer, M. W. Kirschner, and U. K. Laernmli: Peptide mapping by limited proteolysis in sodium dodecyl sulfate and analysis by gel electrophoresis. J. Biol. Chem., 252, 1102-1106 (1977).

18) H. Towbin, T. Staehelin, and J. Gordon: Electrophoretic transfer of proteins from polyacrylamide gels to nitrocellulose sheets: procedure and some applications. Proc. Natn. Acad. Sci. USA, 76, 43504354 (1979).

19) S. Mizuta, R. Yoshinaka, M. Sato, T. Suzuki, and M. Sakaguchi: Immunohistochemical localizationof genetically distinct types of collagen in muscle of kuruma prawn Penaeus japonicus. Comp. Biochem. Physiol., 103B, 917-922 (1992). 